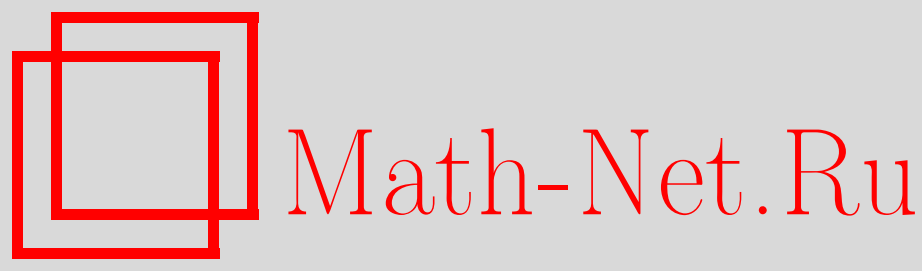

Шунь-Лун Ло, Вэй Сунь, Сепарабельность и запутывание в состояниях систем из трех частей, ТМФ, 2009, том 160, номер 3, 534-544

DOI: https://doi.org/10.4213/tmf6414

Использование Общероссийского математического портала Math-Net.Ru подразумевает, что вы прочитали и согласны с пользовательским соглашением http://www . mathnet.ru/rus/agreement

Параметры загрузки:

IP : 54.210 .77 .194

26 апреля 2023 г., 13:03:00

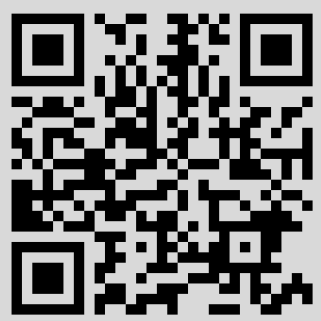




\section{СЕПАРАБЕЛЬНОСТЬ И ЗАПУТЫВАНИЕ В СОСТОЯНИЯХ СИСТЕМ ИЗ ТРЕХ ЧАСТЕЙ}

В то время как классические корреляции можно свободно распределять между несколькими системами, в отношении запутывания и квантовых корреляций это не так. Если квантовая система $S^{a}$ запутана с другой квантовой системой $S^{b}$, то ее запутывание с любой третьей квантовой системой $S^{c}$ не может быть произвольным. В этом состоит свойство моногамности эффекта запутывания. В этом общем утверждении неявно содержится правдоподобное предположение о том, что только запутывание между системами $S^{a}$ и $S^{b}$ налагает связи на запутывание между системой $S^{a}$ и третьей системой $S^{c}$. Показано, что даже классические корреляции между системами $S^{a}$ и $S^{b}$ могут налагать неожиданно строгие ограничения на возможное запутывание между системами $S^{a}$ и $S^{c}$. В частности, идеальные классические корреляции и полное запутывание для систем, состоящих из двух частей, не могут сосуществовать ни в какой системе, состоящей из трех частей. Интуитивное объяснение причин такой моногамности в случае смеси классических и квантовых корреляций может заключаться в том, что система $S^{a}$ имеет определенную способность к корреляции, которую нельзя использовать для установления запутывания с третьей системой (но можно использовать для установления классических корреляций), если она исчерпывается при корреляции с системой $S^{b}$ (классической или квантовой). Это можно интерпретировать как альтернативный вариант свойства моногамности.

Ключевые слова: корреляции, классические состояния, состояния систем из трех частей, запутывание, сепарабельность.

\section{1. ВВЕДЕНИЕ}

Корреляции остаются постоянным предметом исследований в активно развивающейся в последнее время области квантовой теории информации [1]. Общее характеристическое свойство квантовой информации, существенно отличающее ее от классической информации, состоит в том, что первая подчиняется нескольким запрещающим теоремам, таким как теоремы о невозможности клонирования [2]-[5],

*Academy of Mathematics and Systems Science, Chinese Academy of Sciences, Beijing, People's Republic of China. E-mail: luosl@amt.ac.cn

${ }^{\dagger}$ Department of Mathematics and Statistics, Concordia University, Montreal, Quebec, Canada 
невозможности удаления [6], невозможности распространения [7], [8], невозможности сигнализации [9], [10], а также теоремы о моногамности [11]-[15].

Как показали многие авторы [11]-[15], так называемая моногамность запутывания представляет собой следующее явление: факт запутывания квантовой системы с другой налагает ограничения на способность системы к запутыванию с третьей системой. Таким образом, поддерживается своеобразный баланс обмена между запутыванием для различных квантовых систем, состоящих из двух частей и имеющих общую часть. Совсем другая ситуация в случае классических корреляций, которые можно свободно распределять на много систем. Имея это в виду, можно поддаться искушению и предположить, что если две квантовые системы коррелированы только классически, то одна из них могла бы находиться в произвольном запутывании с третьей системой, аналогично тому, как это в общем случае позволено для систем, состоящих из двух частей. Мы покажем, что это иллюзия. Конкретно мы рассмотрим следующий вопрос. Предположим, что квантовая система $S^{a}$ коррелирована с квантовой системой $S^{b}$ только классически. Ограничивают ли тогда хоть как-то эти корреляции запутывание между системой $S^{a}$ и некоторой третьей квантовой системой $S^{c}$ ? Как мы увидим, запутывание между системами $S^{a}$ и $S^{c}$ даже может оказаться запрещенным в силу некоторых классических корреляций между $S^{a}$ и $S^{b}$.

Для решения сформулированной выше проблемы в разделе 2 тщательно определены понятия классических корреляций и запутывания. Основной результат, который заключается в том, что некоторый тип классических корреляций между двумя системами запрещает их возможное запутывание с третьей квантовой системой, установлен в разделе 3. Кроме того, мы даем характеризацию сепарабельных состояний в терминах идеально коррелированных классических состояний, что играет определяющую роль в нашем подходе. Наконец, в разделе 4 приведены некоторые замечания. Чтобы исключить сложности технического плана, мы рассматриваем только конечные размерности.

\section{2. СЕПАРАБЕЛЬНЫЕ СОСТОЯНИЯ, КЛАССИЧЕСКИЕ СОСТОЯНИЯ И БЛИЗНЕЦОВЫЕ СОСТОЯНИЯ}

Рассмотрим составную квантовую систему $S^{a} \otimes S^{b}$, состоящую из двух подсистем $S^{a}$ и $S^{b}$. Ее состояние $\rho^{a b}$ называется сепарабельным, если его можно представить в виде

$$
\rho^{a b}=\sum_{i} p_{i} \rho_{i}^{a} \otimes \rho_{i}^{b}
$$

для некоторого вероятностного распределения $\left\{p_{i}\right\}$ и локальных состояний $\left\{\rho_{i}^{a}\right\}$ для части $a$ и локальных состояний $\left\{\rho_{i}^{b}\right\}$ для части $b$. В противном случае состояние называется запутанным [16]. Такой формализм, соотносящий сепарабельность и запутывание, является прототипом для многих исследований в области квантовой теории информации и служит отправной точкой в настоящей работе. Однако этот исключительно важный и концептуально ясный формализм имеет печальную славу из-за сложностей, возникающих при работе с ним, связанных с неединственностью представления (1). Например, за исключением некоторых специальных случаев, как 
правило, не представляется возможным определить, является ли состояние общего вида для системы, состоящей из двух частей, сепарабельным [17].

Нам потребуется еще одно, связанное с предыдущим, но отличное от него понятие, играющее ключевую роль в установлении нашего основного результата. Состояние $\rho^{a b}$ системы, состоящей из двух частей, называется классическим (относительно корреляций), если существуют локальные фон-неймановские измерения $\left\{\Pi_{i}^{a}\right\}$ и $\left\{\Pi_{j}^{b}\right\}$, выражаемые полными одномерными ортогональными проекциями для частей $a$ и $b$, при которых состояние $\rho^{a b}$ инвариантно при совместном измерении $\Pi=\left\{\Pi_{i}^{a} \otimes \Pi_{j}^{b}\right\}$ в том смысле, что

$$
\rho^{a b}=\Pi\left(\rho^{a b}\right),
$$

где состояние П $\left(\rho^{a b}\right)$ после измерения определяется как

$$
\Pi\left(\rho^{a b}\right):=\sum_{i, j} \Pi_{i}^{a} \otimes \Pi_{j}^{b} \rho^{a b} \Pi_{i}^{a} \otimes \Pi_{j}^{b} .
$$

В противном случае состояние $\rho^{a b}$ является истинно квантовым (относительно корреляций). Такая классификация корреляций для состоящих из двух частей систем, в отличие от стандартной парадигмы, соотносящей сепарабельность и запутывание, ухватывает интуитивную идею о том, что для классического объекта измерение можно произвести так, что оно не изменит объект, тогда как для квантового объекта измерение обычно приводит к изменению рассматриваемого объекта, кроме случаев, когда объект оказывается классическим [18].

Замечательный факт состоит в том, что измерения, участвующие в приведенном выше определении, являются, по существу, единственными. Пусть спектральные разложения маргинальных состояний $\rho^{a}:=\operatorname{tr}_{b} \rho^{a b}$ (частичный след) и $\rho^{b}:=\operatorname{tr}_{a} \rho^{a b}$ имеют вид $\rho^{a}=\sum_{i} p_{i}^{a} \Pi_{i}^{a}$ и $\rho^{b}=\sum_{j} p_{j}^{b} \Pi_{j}^{b}$. Тогда следующие утверждения эквивалентны [18]:

1) $\rho^{a b}$ является классическим состоянием;

2) $\rho^{a b}$ коммутирует с каждым $\Pi_{i}^{a} \otimes \Pi_{j}^{b}$;

3) $\rho^{a b}$ можно представить как

$$
\rho^{a b}=\sum_{i, j} p_{i j} \Pi_{i}^{a} \otimes \Pi_{j}^{b}
$$

для некоторого вероятностного распределения $\left\{p_{i j}\right\}$ по двум переменным (некоторые из $p_{i j}$ могут быть равны нулю). Более того, в данных обстоятельствах мы имеем $p_{i}^{a}=\sum_{j} p_{i j}$ и $p_{j}^{b}=\sum_{i} p_{i j}$. Таким образом, $\rho^{a b}$ и классическое вероятностное распределение $\left\{p_{i j}\right\}$ по двум переменным можно в действительности отождествить канонически, и при этом ясно, что в классическом состоянии отсутствуют квантовые корреляции. Более того, очевидно, что классическое состояние является сепарабельным, но не наоборот.

Для любого состояния $\rho^{a b}$ в системе из двух частей состояние $\Pi\left(\rho^{a b}\right)$ после измерения всегда является классическим состоянием для любых полных проективных измерений $\left\{\Pi_{i}^{a}\right\}$ и $\left\{\Pi_{j}^{b}\right\}$ (даже если они не являются проекторами на собственные векторы для редуцированных состояний $\left.\rho^{a b}\right)$. Это конечное состояние возникает 
вследствие декогеренции, обусловленной измерением П. Недавно было показано, что классические состояния являются единственными состояниями, для которых корреляции допускают локальное распространение (broadcast) [8].

Особенно интересным примером классических состояний являются состояния, для которых $p_{i j}=p_{i} \delta_{i j}$. В этом случае два маргинальных распределения вероятностей идеально коррелированы и мы получаем

$$
\rho^{a b}=\sum_{i} p_{i} \Pi_{i}^{a} \otimes \Pi_{i}^{b}
$$

Такое состояние в системе из двух частей можно интуитивно называть классическим близнецовым состоянием, и оно сыграет ключевую роль при получении нашего основного результата. Заметим, что любое чистое состояние, заданное в виде произведения, является классическим близнецовым состоянием, в то время как любое заданное в виде произведения состояние, в котором по крайней мере одно из двух маргинальных состояний смешано, не может быть классическим близнецовым состоянием. Мы покажем, что классическое близнецовое состояние обладает любопытным свойством: хотя составная система $S^{a} \otimes S^{b}$ в таком состоянии может быть запутанной с другой системой, ни $S^{a}$, ни $S^{b}$ не может быть запутана ни с какой другой системой.

Аналогичным образом можно также ввести следующее определение: состояние $\rho^{a b}$ является классически-квантовым, если существует полное локальное измерение $\left\{\Pi_{i}^{a}\right\}$, не возмущающее состояние $\rho^{a b}$, т.е. $\Pi^{a}\left(\rho^{a b}\right)=\rho^{a b}$. Здесь

$$
\Pi^{a}\left(\rho^{a b}\right):=\sum_{i} \Pi_{i}^{a} \otimes \mathbf{1}^{b} \rho^{a b} \Pi_{i}^{a} \otimes \mathbf{1}^{b}
$$

- конечное состояние после измерения $\Pi^{a}:=\left\{\Pi_{i}^{a} \otimes \mathbf{1}^{b}\right\}$, a $\mathbf{1}^{b}$ - единичный оператор для $S^{b}$. Очевидно, что класс классически-квантовых состояний включает класс классических состояний и входит в класс сепарабельных состояний. Более того, мы имеем следующие эквивалентные утверждения:

1) $\rho^{a b}$ является классически-квантовым состоянием;

2) $\rho^{a b}$ коммутирует с каждым $\Pi_{i}^{a} \otimes \mathbf{1}^{b}$ (здесь $\left\{\Pi_{i}^{a}\right\}$ - одномерные проекторы на собственные состояния для $\left.\rho^{a}:=\operatorname{tr}_{b} \rho^{a b}\right)$;

3) $\rho^{a b}$ можно представить как

$$
\rho^{a b}=\sum_{i} p_{i} \Pi_{i}^{a} \otimes \rho_{i}^{b}
$$

для некоторого распределения вероятностей $\left\{p_{i}\right\}$ и локальных состояний $\rho_{i}^{b}$ для части $b$.

Некоторые замечательные свойства классически-квантовых состояний заключаются в том, что они удовлетворяют супераддитивности косой информации Вигнера-Янасе-Дайсона [19], а их квантовые диссонансы, введенные в работе [20], обращаются в нуль. 


\section{3. СЕПАРАБЕЛЬНОСТЬ, КЛАССИЧНОСТЬ И ЗАПУТЫВАНИЕ}

Теперь мы можем сформулировать наш основной результат.

Теорема. Любое сепарабельное состояние $\rho^{a b}$ системы, состоящей из двух частей, $S^{a} \otimes S^{b}$, можно расширить до классического близнецового состояния (см. (3)) в том смысле, что существует такое классическое близнецовое состояние $\rho^{\text {АВ }}$ системи $S^{A} \otimes S^{B}:=S^{a} \otimes S^{a^{\prime}} \otimes S^{b^{\prime}} \otimes S^{b}$ (здесъ $S^{a^{\prime}}$ и $S^{b^{\prime}}$ - две подчиненные системь, а $\left.S^{A}:=S^{a} \otimes S^{a^{\prime}}, S^{B}:=S^{b^{\prime}} \otimes S^{b}\right)$, что $\rho^{a b}=\operatorname{tr}_{a^{\prime} b^{\prime}} \rho^{A B}$. Более того, если $\rho^{A B c}$ - произвольное состояние системы из трех частей $S^{A} \otimes S^{B} \otimes S^{c}$ такое, что $\rho^{A B}=\operatorname{tr}_{c} \rho^{A B c}$, то система $S^{a}$ не может быть запутана с системой $S^{c}$, m.е. состояние $\rho^{a c}:=\operatorname{tr}_{a^{\prime} \text { в }} \rho^{A B c}$ является сепарабельным. Аналогично состояние $\rho^{b c}:=\operatorname{tr}_{A b^{\prime}} \rho^{A B c}$ также является сепарабельным.

На основании сформулированного выше результата можно заключить, что хотя системы $S^{a}$ и $S^{b}$ не являются запутанными, любая из них по-прежнему не может быть запутана ни с какой третьей системой $S^{c}$, коль скоро состояние $\rho^{a b}$ выводится из классического близнецового состояния, которое, в свою очередь, является маргинальным состоянием, полученным из состоящей из трех частей системы посредством вычисления следа по системе $S^{c}$. В частности, если $\rho^{a b}$ само является классическим близнецовым состоянием, то система $S^{a}$ не может быть запутана ни с какой другой системой $S^{c}$, т.е. состояние $\rho^{a c}$ является сепарабельным. Ясно, что и состояние $\rho^{b c}$ также является сепарабельным. Более того, пусть $S\left(\rho^{a b} \mid \rho^{b}\right):=$ $S\left(\rho^{a b}\right)-S\left(\rho^{b}\right)-$ квантовая условная энтропия (здесь $S\left(\rho^{a b}\right)=-\operatorname{tr} \rho^{a b} \ln \rho^{a b}-$ квантовая энтропия) [21], [22]. Тогда ясно, что $S\left(\rho^{a b} \mid \rho^{b}\right)=0$, когда $\rho^{a b}$ является классическим близнецовым состоянием. С другой стороны, на основании следующей эквивалентной формулировки сильной субаддитивности квантовой энтропии [23]:

$$
S\left(\rho^{a b} \mid \rho^{b}\right)+S\left(\rho^{a c} \mid \rho^{c}\right) \geqslant 0,
$$

мы заключаем, что $S\left(\rho^{a c} \mid \rho^{c}\right) \geqslant 0$. Это неравенство резко контрастирует с тем фактом, что для чисто запутанного состояния условная энтропия всегда отрицательна.

Следует подчеркнуть, что в приведенной выше теореме нельзя избавиться от условия о вложении состояния $\rho^{a b}$ в классическое близнецовое состояние $\rho^{A B}$, которое, в свою очередь, является маргинальным состоянием $\rho^{A B c}$ системы, состоящей из трех частей. В качестве примера рассмотрим состояние $\rho^{a b c}=\rho^{a c} \otimes \rho^{b}$, когда $\rho^{a c}$ является максимально запутанным состоянием между системами $S^{a}$ и $S^{c}$. Ясно, что состояние $\rho^{a b}=\operatorname{tr}_{c} \rho^{a b c}=\rho^{a} \otimes \rho^{b}$ является сепарабельным, однако система $S^{a}$ запутана с $S^{c}$. Важно, что согласно сформулированной выше теореме в этом случае состояние $\rho^{a b c}$ не может быть вложено в состояние $\rho^{A B c}$ состоящей из трех частей системы, так что $\rho^{A B}=\operatorname{tr}_{c} \rho^{A B c}$ является классическим близнецовым состоянием, однако состояние $\rho^{a c}$ является запутанным.

Чтобы продемонстрировать некоторые интуитивные основания теоремы, рассмотрим частный случай, когда $\rho^{a b}$ уже является классическим близнецовым состоянием, определенным в (3). В этом случае квантовая взаимная информация [21], [22]

$$
\mathcal{I}\left(\rho^{a b}\right):=S\left(\rho^{a}\right)+S\left(\rho^{b}\right)-S\left(\rho^{a b}\right)
$$


оказывается равной $S\left(\rho^{a}\right)$. Таким образом, полный корреляционный ресурс в маргинальном состоянии $\rho^{a}$, который количественно выражается посредством величины $S\left(\rho^{a}\right)$, полностью использован в корреляциях между системами $S^{a}$ и $S^{b}$, и, следовательно, разумно полагать, что система $S^{a}$ не обладает никакой остаточной способностью к корреляциям с другой системой квантово-механическим образом. Однако эта система еще может быть коррелирована с другой системой классически. Например, возьмем в качестве $S^{b}$ и $S^{c}$ копии системы $S^{a}$ и положим

$$
\rho^{a b c}:=\sum_{i} p_{i} \Pi_{i}^{a} \otimes \Pi_{i}^{b} \otimes \Pi_{i}^{c}
$$

тогда редуцированные состояния системы из трех частей имеют вид

$$
\rho^{a b}=\sum_{i} p_{i} \Pi_{i}^{a} \otimes \Pi_{i}^{b}, \quad \rho^{a c}=\sum_{i} p_{i} \Pi_{i}^{a} \otimes \Pi_{i}^{c}
$$

а система $S^{a}$ идеально коррелирована как с $S^{b}$, так и с $S^{c}$. Эти корреляции, конечно, являются классическими.

Формально теорема напоминает свойство моногамии (хотя и довольно сильно отличается от него по своей природе) некоторых состояний систем, состоящих из трех частей: если две системы полностью запутаны в состоянии $\left|\psi^{a b}\right\rangle=\sum_{i} \lambda_{i}\left|\psi_{i}^{a}\right\rangle \otimes\left|\psi_{i}^{b}\right\rangle$ (вид Шмидта), то система $S^{a}$ не может быть запутана с системой $S^{c}[11]$. Здесь $S^{a}$ и $S^{b}$ идеально коррелированы как в состоянии $\left|\psi^{a b}\right\rangle$, так и в состоянии $\rho^{a b}$ (если $\rho^{a b}$ является классическим близнецовым состоянием), но по принципиально различным причинам: корреляции в состоянии $\rho^{a b}$ являются чисто классическими, в то время как корреляции в $\left|\psi^{a b}\right\rangle$ - чисто квантовыми. Однако и те и другие корреляции запрещают запутанность системы $S^{a}$ с системой $S^{c}$. Более того, из теоремы также следует, что два упомянутых выше типа (классические и квантовые) предельных идеальных корреляций не могут сосуществовать для состояния системы из трех частей в том смысле, что не существует такого состояния $\rho^{a b c}$, что $\rho^{a b}$ является классическим близнецовым состоянием, а $\rho^{a c}$ - полностью запутанным состоянием.

Для доказательства теоремы заметим сначала, что, как показано в работе [24], любое сепарабельное состояние $\rho^{a b}$ можно расширить до классического состояния, и, следовательно, чтобы доказать первое утверждение теоремы, можно без потери общности предположить, что состояние $\rho^{a b}$ уже является классическим, т.е. $\rho^{a b}$ имеет вид (2).

Пусть теперь $S^{a^{\prime}}$ и $S^{b^{\prime}}$ - копии систем $S^{b}$ и $S^{a}$ соответственно (обратим внимание на обратный порядок), а $\left\{\Pi_{j}^{a^{\prime}}\right\}$ и $\left\{\Pi_{i}^{b^{\prime}}\right\}-$ копии систем $\left\{\Pi_{j}^{b}\right\}$ и $\left\{\Pi_{i}^{a}\right\}$ соответственно. Если положить $\mathbf{k}:=(i, j)$,

$$
\Pi_{\mathbf{k}}^{A}:=\Pi_{i}^{a} \otimes \Pi_{j}^{a^{\prime}}, \quad \Pi_{\mathbf{k}}^{B}:=\Pi_{i}^{b^{\prime}} \otimes \Pi_{j}^{b},
$$

и определить состояние $\rho^{A B}$ как

$$
\rho^{A B}:=\sum_{\mathbf{k}} p_{\mathbf{k}} \Pi_{\mathbf{k}}^{A} \otimes \Pi_{\mathbf{k}}^{B}
$$


то ясно, что $\rho^{A B}$ - это классическое близнецовое состояние для расширенной системы $S^{A} \otimes S^{B}=S^{a} \otimes S^{a^{\prime}} \otimes S^{b^{\prime}} \otimes S^{b}$, причем

$$
\rho^{a b}=\operatorname{tr}_{a^{\prime} b^{\prime}} \rho^{A B} .
$$

Этим завершается доказательство первого утверждения теоремы.

Для доказательства второго утверждения теоремы приведем простую алгебраическую лемму.

ЛЕмма. Если $M$ и $N$-две неотрицательно определенные эрмитовы матрицы maкue, чmo $\operatorname{tr} M N=0$, mo $M N=0$.

Чтобы показать это, предположим, что матрица $N$ обратима. Тогда, поскольку $\operatorname{tr} M N=\operatorname{tr} N^{1 / 2} M N^{1 / 2}=0$ и $N^{1 / 2} M N^{1 / 2}$ является неотрицательно определенной матрицей, получаем $N^{1 / 2} M N^{1 / 2}=0$. Умножая обе части этого равенства на $N^{-1 / 2}$ и $N^{1 / 2}$ соответственно слева и справа, заключаем, что $M N=0$. Если $N$ не обратима, то мы можем привлечь соображения непрерывности и прийти к тому же заключению.

Теперь из определения состояния $\rho^{A B}$ при $\mathbf{k} \neq \mathbf{k}^{\prime}$ получаем

$$
\operatorname{tr}\left(\Pi_{\mathbf{k}}^{A} \otimes \Pi_{\mathbf{k}^{\prime}}^{B} \otimes \mathbf{1}^{c}\right) \rho^{A B c}=\operatorname{tr}\left(\Pi_{\mathbf{k}}^{A} \otimes \Pi_{\mathbf{k}^{\prime}}^{B}\right) \rho^{A B}=0 .
$$

Отсюда с учетом приведенной выше леммы при $\mathbf{k} \neq \mathbf{k}^{\prime}$ получаем

$$
\left(\Pi_{\mathbf{k}}^{A} \otimes \Pi_{\mathbf{k}^{\prime}}^{B} \otimes \mathbf{1}^{c}\right) \rho^{A B c}=0 .
$$

Поэтому, используя $\sum_{\mathbf{k}^{\prime}} \Pi_{\mathbf{k}^{\prime}}^{A}=\mathbf{1}^{A}$, находим

$$
\left(\mathbf{1}^{A} \otimes \Pi_{\mathbf{k}}^{B} \otimes \mathbf{1}^{c}\right) \rho^{A B c}=\left(\left(\sum_{\mathbf{k}^{\prime}} \Pi_{\mathbf{k}^{\prime}}^{A}\right) \otimes \Pi_{\mathbf{k}}^{B} \otimes \mathbf{1}^{c}\right) \rho^{A B c}=\left(\Pi_{\mathbf{k}}^{A} \otimes \Pi_{\mathbf{k}}^{B} \otimes \mathbf{1}^{c}\right) \rho^{A B c},
$$

и аналогично, используя $\sum_{\mathbf{k}^{\prime}} \Pi_{\mathbf{k}^{\prime}}^{B}=\mathbf{1}^{B}$, получаем

$$
\left(\Pi_{\mathbf{k}}^{A} \otimes \mathbf{1}^{B} \otimes \mathbf{1}^{c}\right) \rho^{A B c}=\left(\Pi_{\mathbf{k}}^{A} \otimes\left(\sum_{\mathbf{k}^{\prime}} \Pi_{\mathbf{k}^{\prime}}^{B}\right) \otimes \mathbf{1}^{c}\right) \rho^{A B c}=\left(\Pi_{\mathbf{k}}^{A} \otimes \Pi_{\mathbf{k}}^{B} \otimes \mathbf{1}^{c}\right) \rho^{A B c} .
$$

Комбинируя соотношения (5) и (6), получаем

$$
\left(\mathbf{1}^{A} \otimes \Pi_{\mathbf{k}}^{B} \otimes \mathbf{1}^{c}\right) \rho^{A B c}=\left(\Pi_{\mathbf{k}}^{A} \otimes \mathbf{1}^{B} \otimes \mathbf{1}^{c}\right) \rho^{A B c} .
$$

Для сопряженного (7) уравнения мы также получаем

$$
\rho^{A B c}\left(\mathbf{1}^{A} \otimes \Pi_{\mathbf{k}}^{B} \otimes \mathbf{1}^{c}\right)=\rho^{A B c}\left(\Pi_{\mathbf{k}}^{A} \otimes \mathbf{1}^{B} \otimes \mathbf{1}^{c}\right) .
$$

Эти два соотношения исключительно важны для последующих рассуждений. На самом деле они являются переформулировкой допущения о том, что системы $S^{A}$ и $S^{B}$ идеально коррелированы классическим способом.

Для любых наблюдаемых $X^{A}$ и $X^{c}$ для соответствующих систем $S^{A}$ и $S^{c}$ заметим, что $X^{A} \otimes \mathbf{1}^{B} \otimes X^{c}$ коммутирует с $\mathbf{1}^{A} \otimes \Pi_{\mathbf{k}}^{B} \otimes \mathbf{1}^{c}$, а также $\mathbf{1}^{A} \otimes \Pi_{\mathbf{k}}^{B} \otimes \mathbf{1}^{c}=\left(\mathbf{1}^{A} \otimes \Pi_{\mathbf{k}}^{B} \otimes \mathbf{1}^{c}\right)^{2}$. 
Используя свойство цикличности следа, с учетом соотношений (7) и (8), а также равенства $\sum_{\mathbf{k}} \Pi_{\mathbf{k}}^{B}=\mathbf{1}^{B}$, получаем

$$
\begin{aligned}
\operatorname{tr}\left(X^{A} \otimes X^{c}\right) \rho^{A c} & =\operatorname{tr}\left(X^{A} \otimes \mathbf{1}^{B} \otimes X^{c}\right) \rho^{A B c}= \\
& =\operatorname{tr}\left(X^{A} \otimes \mathbf{1}^{B} \otimes X^{c}\right)\left(\mathbf{1}^{A} \otimes\left(\sum_{\mathbf{k}} \Pi_{\mathbf{k}}^{B}\right) \otimes \mathbf{1}^{c}\right) \rho^{A B c}= \\
& =\sum_{\mathbf{k}} \operatorname{tr}\left(\mathbf{1}^{A} \otimes \Pi_{\mathbf{k}}^{B} \otimes \mathbf{1}^{c}\right)\left(X^{A} \otimes \mathbf{1}^{B} \otimes X^{c}\right)\left(\mathbf{1}^{A} \otimes \Pi_{\mathbf{k}}^{B} \otimes \mathbf{1}^{c}\right) \rho^{A B c}= \\
& =\sum_{\mathbf{k}} \operatorname{tr}\left(\mathbf{1}^{A} \otimes \Pi_{\mathbf{k}}^{B} \otimes \mathbf{1}^{c}\right)\left(X^{A} \otimes \mathbf{1}^{B} \otimes X^{c}\right)\left(\Pi_{\mathbf{k}}^{A} \otimes \mathbf{1}^{B} \otimes \mathbf{1}^{c}\right) \rho^{A B c}= \\
& =\sum_{\mathbf{k}} \operatorname{tr}\left(X^{A} \otimes \mathbf{1}^{B} \otimes X^{c}\right)\left(\Pi_{\mathbf{k}}^{A} \otimes \mathbf{1}^{B} \otimes \mathbf{1}^{c}\right) \rho^{A B c}\left(\mathbf{1}^{A} \otimes \Pi_{\mathbf{k}}^{B} \otimes \mathbf{1}^{c}\right)= \\
& =\sum_{\mathbf{k}} \operatorname{tr}\left(X^{A} \otimes \mathbf{1}^{B} \otimes X^{c}\right)\left(\Pi_{\mathbf{k}}^{A} \otimes \mathbf{1}^{B} \otimes \mathbf{1}^{c}\right) \rho^{A B c}\left(\Pi_{\mathbf{k}}^{A} \otimes \mathbf{1}^{B} \otimes \mathbf{1}^{c}\right)= \\
& =\sum_{\mathbf{k}} \operatorname{tr}\left(\Pi_{\mathbf{k}}^{A} \otimes \mathbf{1}^{B} \otimes \mathbf{1}^{c}\right)\left(X^{A} \otimes \mathbf{1}^{B} \otimes X^{c}\right)\left(\Pi_{\mathbf{k}}^{A} \otimes \mathbf{1}^{B} \otimes \mathbf{1}^{c}\right) \rho^{A B c}= \\
& =\sum_{\mathbf{k}} \operatorname{tr}\left(\Pi_{\mathbf{k}}^{A} X^{A} \Pi_{\mathbf{k}}^{A} \otimes \mathbf{1}^{B} \otimes X^{c}\right) \rho^{A B c}= \\
& =\sum_{\mathbf{k}} \operatorname{tr}\left(\Pi_{\mathbf{k}}^{A} X^{A} \Pi_{\mathbf{k}}^{A} \otimes X^{c}\right) \rho^{A c}= \\
& =\sum_{\mathbf{k}} \operatorname{tr}\left(X^{A} \otimes X^{c}\right)\left(\Pi_{\mathbf{k}}^{A} \otimes \mathbf{1}^{c}\right) \rho^{A c}\left(\Pi_{\mathbf{k}}^{A} \otimes \mathbf{1}^{c}\right) .
\end{aligned}
$$

Отсюда

$$
\rho^{A c}=\sum_{\mathbf{k}}\left(\Pi_{\mathbf{k}}^{A} \otimes \mathbf{1}^{c}\right) \rho^{A c}\left(\Pi_{\mathbf{k}}^{A} \otimes \mathbf{1}^{c}\right)
$$

и согласно эквивалентным утверждениям 1-3 (где $A$ играет роль $a$ ), сформулированным в конце раздела 2, можно записать

$$
\rho^{A c}=\sum_{\mathbf{k}} p_{\mathbf{k}} \Pi_{\mathbf{k}}^{A} \otimes \rho_{\mathbf{k}}^{c}
$$

Если положить $\rho_{\mathbf{k}}^{a}=\operatorname{tr}_{a^{\prime}} \Pi_{\mathbf{k}}^{A}$, то мы увидим, что маргинальное состояние

$$
\rho^{a c}=\operatorname{tr}_{a^{\prime}} \rho^{A c}=\sum_{\mathbf{k}} p_{\mathbf{k}}\left(\operatorname{tr}_{a^{\prime}} \Pi_{\mathbf{k}}^{A}\right) \otimes \rho_{\mathbf{k}}^{c}=\sum_{\mathbf{k}} p_{\mathbf{k}} \rho_{\mathbf{k}}^{a} \otimes \rho_{\mathbf{k}}^{c}
$$

является сепарабельным. Это завершает доказательство.

В качестве интересного следствия доказанной теоремы рассмотрим частный случай, когда $\rho^{a b}$ уже является классическим близнецовым состоянием. Тогда согласно теореме ни $S^{a}$, ни $S^{b}$ нельзя запутать ни с какой другой системой (оба редуцированных состояния для систем $S^{a} \otimes S^{c}$ и $S^{b} \otimes S^{c}$ являются сепарабельными), хотя систему с частями $S^{a}$ и $S^{b}$ как целое можно запутать с другой системой! Проиллюстрируем последнее утверждение. Очистим состояние $\rho^{a b}$, определяемое в (3), 
в системе $S^{a} \otimes S^{b} \otimes S^{a^{\prime}} \otimes S^{b^{\prime}}$ следующим образом:

$$
\left|\Psi^{a b a^{\prime} b^{\prime}}\right\rangle=\sum_{i} \sqrt{p_{i}}\left|\psi_{i}^{a}\right\rangle \otimes\left|\psi_{i}^{b}\right\rangle \otimes\left|\psi_{i}^{a^{\prime}}\right\rangle \otimes\left|\psi_{i}^{b^{\prime}}\right\rangle
$$

где $\Pi_{i}^{a}=\left|\psi_{i}^{a}\right\rangle\left\langle\psi_{i}^{a}\left|, \Pi_{i}^{b}=\right| \psi_{i}^{b}\right\rangle\left\langle\psi_{i}^{b}\right|$, а $\left|\psi_{i}^{a^{\prime}}\right\rangle$ и $\left|\psi_{i}^{b^{\prime}}\right\rangle$ являются копиями $\left|\psi_{i}^{a}\right\rangle$ и $\left|\psi_{i}^{b}\right\rangle$ соответственно. Если рассматривать систему $S^{a^{\prime}} \otimes S^{b^{\prime}}$ как систему $S^{c}$, то система $S^{a} \otimes S^{b}$ будет запутана с $S^{c}$ с энтропией запутывания $-\sum_{i} p_{i} \ln p_{i}[25]$. В этом случае можно интуитивно полагать, что именно классические корреляции в системе $S^{a} \otimes S^{b}$ реализуют запутывание с $S^{c}$, т.е. запутывающими объектами из части $S^{a} \otimes S^{b}$ являются не $S^{a}$ или $S^{b}$, а корреляции в них.

\section{4. ОБСУЖДЕНИЕ}

В то время как классические корреляции можно свободно распределять между неограниченным числом систем, хорошо известно, что с запутыванием этого делать нельзя, что иллюстрируется многочисленными моногамными характеристиками запутывания. В свете этого возникает естественный вопрос: что же происходит в смешанном случае, когда классические корреляции поделены между некоторыми системами, а запутывание поделено между другими несколькими системами? Нами получены результаты, проливающие свет на решение этой задачи. В частности, мы выявили интересное свойство корреляций для состоящих из нескольких частей систем в терминах классических близнецовых состояний: в некоторых случаях классические корреляции между двумя квантовыми системами $S^{a}$ и $S^{b}$ исключают всякое запутывание между системой $S^{a}$ (или $S^{b}$ ) и любой другой квантовой системой. Это позволяет получить альтернативную и дополнительную характеризацию моногамности запутывания. Представляет интерес исследование количественных соотношений обмена, связывающих корреляции (как классические, так и квантовые) между системами $S^{a}$ и $S^{b}$ и квантовые корреляции между системами $S^{a}$ и $S^{c}$, а также связанных взаимодействий между классическими и квантовыми корреляциями для состояний систем, состоящих из многих частей.

Мы проиллюстрировали некоторое сходство характеристик запутывания и классических близнецовых состояний и показали, что это связано с проблемой классических аналогов квантового запутывания [26], [27]. В частности, Аллавердян с соавторами [27] открыли классический аналог квантового запутывания для броуновских частиц. Это эффект корреляции между координатами двух броуновских частиц и их усредненными скоростями. Представляет интерес проблема совместности между запутыванием и его классическими аналогами для систем, состоящих из многих частей.

Полученные нами результаты также могут быть полезны при решении задач о маргинальных квантовых состояниях, которая связана с вопросом о том, почему различные состояния в квантовых системах, состоящих из многих частей, являются совместными в том смысле, что они возникают как маргинальные состояния 
большего объединенного состояния. Хорошо известно, что эта задача представляет собой вариант задачи об $N$-представимости, и в общем случае ее трудно решить [28], [29]. Из доказанной теоремы следует, что любое классическое близнецовое состояние несовместно ни с каким запутанным состоянием, т.е. они не могут одновременно оказаться редуцированными маргинальными состояниями системы, состоящей из трех частей. Отсюда сразу же следует результат о невозможности в задаче о квантовом маргинальном состоянии. Однако в классическом случае Воробьев [30] решил задачу совместности семейства случайных переменных в том смысле, что их можно реализовать в одном колмогоровском пространстве вероятностей. Этот результат имеет важные приложения для интерпретации неравенств Белла [31]. Хренников [31] представил логически строгий анализ нарушения неравенств Белла, несовместности семейств случайных переменных и связанных с этим физических проблем вещественности и локальности. Исследование совместности и взаимного влияния различных корреляционных структур, как квантовых, так и классических, лежит в основе теории информации.

Благодарности. Данная работа была поддержана со стороны NSFC (грант № 10771208), Science Fund for Creative Research Groups (грант № 10721101) и NSERC (грант № 311945-2008).

\section{Список литературы}

[1] M. A. Nielsen, I. L. Chuang, Quantum Computation and Quantum Information, Cambridge Univ. Press, Cambridge, 2000.

[2] W. K. Wootters, W. H. Zurek, Nature, 299 (1982), 802-803.

[3] D. Dieks, Phys. Lett. A, 92:6 (1982), 271-272.

[4] H. P. Yuen, Phys. Lett. A, 113:8 (1986), 405-407.

[5] V. Scarani, S. Iblisdir, N. Gisin, Rev. Modern Phys., 77:4 (2005), 1225-1256.

[6] A. K. Pati, S. L. Braunstein, Nature, 404 (2000), 164-165.

[7] H. Barnum, C. Caves, C. Fuchs, R. Jozsa, B. Schumacher, Phys. Rev. Lett., 76:15 (1996), 2818-2821.

[8] M. Piani, P. Horodecki, R. Horodecki, Phys. Rev. Lett., 100:9 (2008), 090502.

[9] M. Piani, M. Horodecki, P. Horodecki, R. Horodecki, Phys. Rev. A, 74:1 (2006), 012305.

[10] L. Masanes, A. Acin, N. Gisin, Phys. Rev. A, 73:1 (2006), 012112.

[11] V. Coffman, J. Kundu, W. K. Wootters, Phys. Rev. A, 61:5 (2000), 052306.

[12] B. M. Terhal, IBM J. Res. Develop., 48:1 (2004), 71-78.

[13] T. J. Osborne, F. Verstraete, Phys. Rev. Lett., 96:22 (2006), 220503.

[14] G. Adesso, F. Illuminati, Phys. Rev. Lett., 99:15 (2007), 150501.

[15] Y.-C. Ou, Phys. Rev. A, 75:3 (2007), 034305.

[16] R. F. Werner, Phys. Rev. A, 40:8 (1989), 4277-4281.

[17] A. Peres, Phys. Rev. Lett., 77:8 (1996), 1413-1415; M. Horodecki, P. Horodecki, R. Horodecki, Phys. Lett. A, 223:1-2 (1996), 1-8; M. Lewenstein, B. Kraus, J. I. Cirac, P. Horodecki, Phys. Rev. A, 62:5 (2000), 052310; H. F. Hofmann, S. Takeuchi, Phys. Rev. A, 68:3 (2003), 032103; O. Gühne, N. Lütkenhaus, Phys. Rev. Lett., 96:17 (2006), 170502.

[18] S. Luo, Phys. Rev. A, 77:2 (2008), 022301.

[19] S. Luo, Q. Zhang, J. Stat. Phys., 131:6 (2008), 1169-1177.

[20] H. Ollivier, W. H. Zurek, Phys. Rev. Lett., 88:1 (2001), 017901; W. H. Zurek, Rev. Modern Phys., 75:3 (2003), 715-775.

[21] A. Wehrl, Rev. Modern Phys., 50:2 (1978), 221-260. 
[22] V. Vedral, Rev. Modern Phys., 74:1 (2002), 197-234.

[23] M. B. Ruskai, J. Math. Phys., 43:9 (2002), 4358-4375.

[24] N. Li, S. Luo, Phys. Rev. A, 78:2 (2008), 024303.

[25] C. H. Bennett, D. P. DiVincenzo, J. A. Smolin, W. K. Wootters, Phys. Rev. A, 54:5 (1996), 3824-3851.

[26] D. Collins, S. Popescu, Phys. Rev. A, 65:3 (2002), 032321.

[27] A. Allahverdyan, A. Khrennikov, Th. M. Nieuwenhuizen, Phys. Rev. A, 72:3 (2005), 032102.

[28] Y.-K. Liu, M. Christandl, F. Verstraete, Phys. Rev. Lett., 98:11 (2007), 110503.

[29] A. J. Coleman, V. I. Yukalov, Reduced Density Matrices. Coulson's Challenge, Lecture Notes in Chemistry, 72, Springer, Berlin, 2000.

[30] Н. Н. Воробьев, ТВП, 7:2 (1962), 153-169.

[31] A. Khrennikov, Bell's inequality: Physics meets Probability, arXiv: 0709.3909, 2007. 